\title{
Screening for primary aldosteronism is underutilised in patients with chronic kidney disease
}

\author{
Karanjeet Chauhan ${ }^{1} \cdot$ Eitan Schachna ${ }^{2} \cdot$ Renata Libianto $^{3,4}$. Jessica Ryan ${ }^{1,5} \cdot$ Holly Hutton $^{2,6} \cdot$ Peter J. Fuller ${ }^{3,4}$. \\ Scott Wilson ${ }^{2,6} \cdot$ Peter G. Kerr ${ }^{1,5}$. Jun Yang ${ }^{1,3,4}$
}

Received: 5 September 2021 / Accepted: 1 February 2022 / Published online: 23 February 2022

(c) The Author(s) 2022

\begin{abstract}
Background Primary aldosteronism (PA) is the most common and potentially curable endocrine cause of secondary hypertension, and carries a worse prognosis than essential hypertension. Despite the high prevalence of hypertension in patients with chronic kidney disease (CKD), the screening rates for primary aldosteronism in CKD are unknown.

Methods In this study, we retrospectively reviewed medical records of 1627 adults who presented to the nephrology clinics of 2 tertiary hospitals in Melbourne, Australia, between 2014 and 2019. In addition to assessing the pattern of screening, we also evaluated patient-specific factors associated with the decision to test for primary aldosteronism. Patients were excluded from the final analysis if they did not have CKD, had an organ transplant, had end stage renal failure, or had insufficient data or follow-up.

Results Of the 600 patients included in the analysis, 234 (39\%) had an indication for screening for primary aldosteronism based on recommendations made by the Endocrine Society. However, only 33 (14\%) were tested. They were younger, had a higher mean systolic blood pressure, better renal function, and lower mean serum potassium than those who were indicated but not screened. Of the 33 screened patients, an elevated aldosterone-to-renin ratio was noted in 8 patients and a diagnosis of primary aldosteronism was made in 4 patients.

Conclusions The screening rate for primary aldosteronism is low in a CKD population, especially in patients who are older, have a lower eGFR and normal serum potassium. The consequences of undiagnosed primary aldosteronism in this select population may be substantial due to the cardiovascular and renal sequelae associated with untreated disease.
\end{abstract}

Jun Yang

Jun.yang@hudson.org.au

1 School of Clinical Sciences, Monash University, Clayton, Australia

2 Central Clinical School, Monash University, Clayton, Australia

3 Department of Endocrinology, Monash Health, Clayton, Australia

4 Endocrine Hypertension Group, Centre for Endocrinology and Metabolism, Hudson Institute of Medical Research, Level 3, Block E, Monash Medical Centre, Clayton Road, Clayton, VIC 3168, Australia

5 Department of Nephrology, Monash Health, Clayton, Australia

6 Department of Nephrology, Alfred Health, Melbourne, Australia 


\section{Graphical abstract}

\section{Is screening for Primary Aldosteronism Underutilised in a Cohort of Patients with Chronic Kidney Disease?}

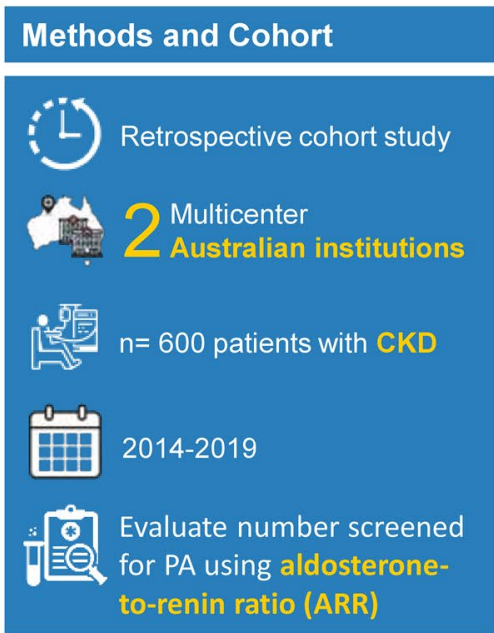

Conclusion: The screening rate for PA is low in a CKD population, especially in patients who are older, have a lower eGFR and normal serum potassium

\section{Findings}

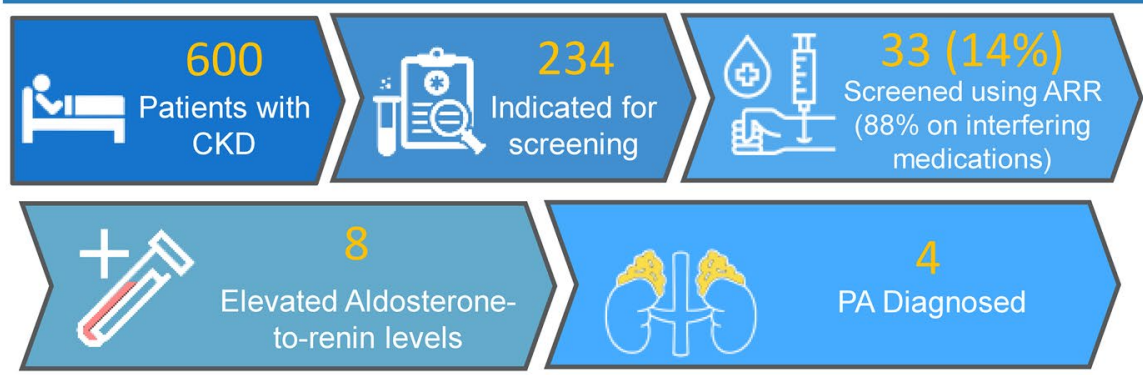

Factors associated with greater screening for Primary Aldosteronism

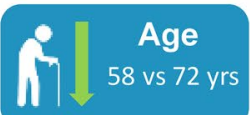

Blood pressure

153 vs $140 \mathrm{mmHg}$

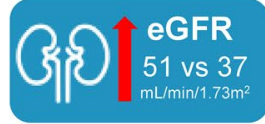

Serum Potassium

4.1 vs $4.5 \mathrm{mEq} / \mathrm{L}$

Keywords Chronic kidney disease $\cdot$ Primary aldosteronism $\cdot$ Hypertension $\cdot$ Hypokalemia $\cdot$ Screening

\section{Introduction}

Hypertension and chronic kidney disease (CKD) are interlinked major public health problems, affecting up to $34 \%$ [1] and 10\% [2] of the Australian population, respectively. Worldwide, hypertension is a significant cause of CKD. In addition, up to $60-90 \%$ of individuals with chronic renal impairment (regardless of aetiology), also have hypertension [3].

The aetiology of hypertension in CKD is multifactorial. A combination of sympathetic activation, increased vascular resistance and sodium retention plays a key role in this process [3]. Poorly controlled hypertension is linked to a rapid deterioration in renal function and a disproportionately high rate of cardiovascular events, which is a leading cause of morbidity and mortality in this patient group [3]. Although the consequences of hypertension in CKD are well known, blood pressure control in this group of patients can be difficult. While the use of antihypertensives reduces morbidity and mortality, up to $40 \%$ of patients have treatment-resistant hypertension defined as inadequately controlled blood pressure (BP) despite the use of three antihypertensive agents or BP controlled on four or more medications [4].
Primary aldosteronism (PA), also called Conn syndrome, is the most common endocrine cause of secondary hypertension and is highly prevalent in patients with treatment-resistant hypertension [5]. PA is characterised by the autonomous production of aldosterone that is not suppressed by salt loading. This is most commonly due to either bilateral adrenal hyperplasia (BAH) or an aldosterone-producing adenoma (APA) $[4,5]$. The autonomous production of aldosterone leads to suppressed renin and an elevated aldosterone-torenin ratio (ARR) which is the standard screening test for PA $[6,7]$. Patients with an elevated ARR subsequently undergo confirmatory testing followed by adrenal venous sampling to differentiate between unilateral disease which can be cured by adrenalectomy, and bilateral disease which can be effectively managed with targeted therapy through mineralocorticoid receptor antagonists $[8,9]$.

PA has long been considered a relatively benign form of hypertension. However, recent evidence has shown that patients with PA are at a 4- to 12-fold increased risk of myocardial infarction, stroke, renal impairment, and poorer quality of life when compared to patients with essential hypertension independent of blood pressure [10-13]. Aldosterone stimulates renal sodium reabsorption and volume expansion which have been found to cause direct podocyte 
injury through persistently increased renal perfusion and glomerular hyperfiltration [14]. Two recent studies demonstrated that the degree of renal dysfunction seen in untreated primary aldosteronism is closely linked to renal haemodynamic adaption to aldosterone excess $[14,15]$. Renal biopsy specimens of 19 patients with unilateral hyperaldosteronism, compared to 22 patients with estimated glomerular filtration rate (eGFR)-matched essential hypertension, demonstrated significantly increased segmental glomerulosclerosis, interstitial fibrosis, and hyalinization of arterioles [16]. Additionally, the hyperfiltration seen in hyperaldosteronism tends to mask the severity of underlying renal damage. This is evidenced by the paradoxical drop in eGFR following PA treatment despite improvements in arterial blood pressure and the degree of proteinuria [14-17]. Targeted treatments have also been shown to reduce all-cause mortality (HR, 0.23 [CI 0.13-0.26]) [18], renal impairment [19], and cardiovascular risk [20], and to increase quality-adjusted life years [21].

The prevalence of PA among hypertensive patients was previously accepted at approximately $1 \%$ [22]. However, recent international literature has found it to be significantly higher at $3-13 \%$ in primary care and up to $30 \%$ in referral centres [23]. Despite the high prevalence of PA, the potential for targeted treatment and published guidelines on circumstances where PA testing is warranted (Fig. 1 [24]), the rates of screening for PA are low [25, 26]. A comparison of the recommended indications for screening and preferred testing modalities of different societies has been shown in Table 1 [27].
Fig. 1 Endocrine society recommendations for PA case detection

\author{
- Sustained blood pressure (BP) above $150 / 100 \mathrm{mmHg}$ on each of three measurements obtained on \\ different days, with hypertension ( $B P>140 / 90 \mathrm{mmHg}$ ) resistant to three conventional \\ antihypertensive drugs (including a diuretic), or controlled BP $(<140 / 90 \mathrm{mmHg}$ ) on four or more \\ antihypertensive drugs \\ - Hypertension and spontaneous or diuretic-induced hypokalaemia \\ - Hypertension and adrenal incidentaloma \\ - Hypertension and sleep apnoea \\ - Hypertension and a family history of early onset hypertension or cerebrovascular accident at a young \\ age ( $<40$ years) \\ - All hypertensive first-degree relatives with primary aldosteronism
}

Table 1 Recommended indications for screening and testing modalities of different societies

\begin{tabular}{|c|c|c|c|c|c|}
\hline \multirow[t]{2}{*}{ Recommendations } & \multicolumn{5}{|c|}{ Specialist guideline } \\
\hline & ES & JES & SIIA & POL & SFE/SFHTA \\
\hline \multicolumn{6}{|l|}{ Screening } \\
\hline Hypertension & $\mathrm{R}$ & $\mathrm{R}$ & $\mathrm{R}$ & $\mathrm{R}$ & $\mathrm{R}$ \\
\hline BP Cut-off (mmHg) & $150 / 100$ & $140 / 90$ & $160 / 90$ & $160 / 100$ & $180 / 110$ \\
\hline Resistant Hypertension & $\mathrm{R}$ & $\mathrm{R}$ & $\mathrm{R}$ & $\mathrm{R}$ & $\mathrm{R}$ \\
\hline Hypertension with Adrenal incidentaloma & $\mathrm{R}$ & NM & $\mathrm{R}$ & $\mathrm{R}$ & $\mathrm{R}$ \\
\hline Hypokalaemia & $\mathrm{R}$ & $\mathrm{R}$ & $\mathrm{R}$ & $\mathrm{R}$ & $\mathrm{R}$ \\
\hline $\begin{array}{l}\text { Family history of early-onset hypertension or } \\
\text { cerebrovascular accident at a young age }\end{array}$ & $\mathrm{R}(<40$ years $)$ & NM & $\mathrm{R}(<50$ years $)$ & $\mathrm{R}(<40$ years $)$ & NM \\
\hline First degree relatives of patients with PA & $\mathrm{R}$ & NM & $\mathrm{R}$ & $\mathrm{R}$ & NM \\
\hline Sleep apnoea & $\mathrm{R}$ & NM & NM & $\mathrm{R}$ & NM \\
\hline Screening test of choice & \multicolumn{5}{|c|}{ Aldosterone-to-renin ratio (ARR) } \\
\hline \multicolumn{6}{|l|}{ Confirmatory testing } \\
\hline Saline infusion test & $\mathrm{R}$ & $\mathrm{R}$ & NM & $\mathrm{R}$ & $\mathrm{R}$ \\
\hline Oral sodium loading test & $\mathrm{R}$ & $\mathrm{R}$ & NM & $\mathrm{R}$ & $\mathrm{R}$ \\
\hline Urinary aldosterone (nmol/24 h) & $\mathrm{R}$ & $\mathrm{R}$ & NM & NM & NM \\
\hline Captopril challenge test & $\mathrm{R}$ & $\mathrm{R}$ & NM & NM & $\mathrm{R}$ \\
\hline Fludrocortisone suppression test & $\mathrm{R}$ & NM & NM & $\mathrm{R}$ & NM \\
\hline
\end{tabular}

ES the Endocrine Society, JES the Japan Endocrine Society, SIIA the Italian Society of Hypertension (Societa' Italiana Dell' Ipertensione Arteriosa), POL Poland, SFE/SFHTA the French Endocrinology Society/French Hypertension Society, $R$ recommended, $N M$ not mentioned 
Lastly, PA testing is not specifically discussed in the current guidelines for management of hypertension in CKD and optimal strategies for diagnosing $\mathrm{PA}$ in $\mathrm{CKD}$ remain unknown even though targeted PA treatment is reno-protective [28-30]

Population-based studies of health systems in Canada and the United States have found that the screening rates for PA were less than $3 \%$ of those for whom it was recommended $[25,31]$. However, similar studies have not been performed to assess screening rates for PA in patients with CKD.

We therefore set out to determine the proportion of patients with CKD attending two large tertiary nephrology outpatient clinics in Victoria, Australia, who had indications for PA screening and to assess the proportion that were appropriately screened. We also aimed to identify patientspecific factors associated with the decision by physicians to screen for PA.

\section{Materials and methods}

\section{Study design and participants}

In this retrospective cohort study, we assessed the medical records of 1627 consecutive patients who attended the nephrology clinics at Monash Health and Alfred Health, two major tertiary health services in Victoria, Australia, from January 2014 to April 2019.

Scanned medical records were manually assessed and data on the aetiology of CKD, diagnosis of hypertension (year, antihypertensive medication, and blood pressure measurements over 3 visits), smoking history, alcohol consumption history and comorbidities (including coronary artery disease, stroke, atrial fibrillation and left ventricular hypertrophy) were recorded. Biochemical data were extracted from the pathology databases of the respective health services.

Patients were excluded if their eGFR was greater than $60 \mathrm{~mL} / \mathrm{min} / 1.73 \mathrm{~m}^{2}$ or if they had less than 3 blood pressure measurements during follow-up visits. We also excluded patients with end-stage renal failure defined by eGFR $<15 \mathrm{~mL} / \mathrm{min} / 1.73 \mathrm{~m}^{2}$ and organ transplants due to the complexities associated with their management, including multiple medications which can affect aldosterone and renin status.

Approval for this project was obtained from the Human Research Ethics Committees of the respective healthcare services.

\section{Laboratory measurements}

In general, clinical practice in the various pathology laboratories was to collect blood samples for aldosterone, renin and their ratio in the morning between 0800 and 1000 AM. Plasma aldosterone and direct renin concentrations were measured using chemiluminescent immunoassays on a DiaSorin Liaison analyser (DiaSorin, Saluggia, Italy). An ARR value $\geq 70 \mathrm{pmol} / \mathrm{mU}$ (20 ng/dL per $\mathrm{ng} / \mathrm{ml} / \mathrm{h}$ ) was considered positive where plasma aldosterone concentration was measured in pmol/L and direct renin concentration in $\mathrm{mU} / \mathrm{L}$. This is equivalent to approximately $20 \mathrm{ng} / \mathrm{dL}$ per $\mathrm{ng} /$ $\mathrm{mL} / \mathrm{h}$ when aldosterone is measured in $\mathrm{ng} / \mathrm{dL}$ and renin is measured as plasma renin activity in $\mathrm{ng} / \mathrm{mL} / \mathrm{h}$ [32]. However, plasma renin activity is no longer routinely measured in most centres including our own [33].

The standard recommendation following a positive screening test was to conduct confirmatory testing using the saline suppression test. Two litres of saline was infused over $4 \mathrm{~h}$ and the diagnosis of PA was confirmed if the plasma aldosterone concentration remained $\geq 140 \mathrm{pmol} / \mathrm{L}(5.0 \mathrm{ng} /$ $\mathrm{dL})$ after a recumbent test or $\geq 170 \mathrm{pmol} / \mathrm{L}(6.1 \mathrm{ng} / \mathrm{dL})$ after a seated test [24].

\section{Statistical analysis}

The Kolmogorov-Smirnov test was used to assess data for normality. Continuous variables were expressed as median (interquartile range), and between-group differences were compared using the nonparametric (Mann Whitney U) test. Categorical variables were expressed as count (percentage) and comparisons between groups were analysed using the Chi-square test. A two-sided p-value of $<0.05$ was considered statistically significant within a $95 \%$ confidence interval. IBM SPSS Statistics for Windows version 26 (IBM Corp., Armonk, N.Y., USA) was used to perform all statistical analyses.

\section{Results}

\section{Patient demographics and clinical characteristics}

Of the 1627 patients who attended the nephrology clinics, 1027 were excluded from the final analysis (Fig. 2). They included 333 patients who did not have CKD, 310 who had insufficient follow-up or less than three blood pressure measurements after their initial presentation, 158 patients who underwent an organ transplant. 12 who had a pre-existing diagnosis of PA prior to attending the nephrology clinic and 214 with end-stage renal disease or on dialysis.

Six hundred patients with CKD were included in the final analysis. Based on current PA screening recommendations made by the Endocrine Society (Fig. 2), 234 of the 600 patients $(39 \%)$ met at least one criterion for PA testing while $52(8.6 \%)$ and $5(0.8 \%)$ met two and three criteria, respectively. Demographics and baseline data of the two groups 
Fig. 2 Flowchart of the selection criteria and inclusion process

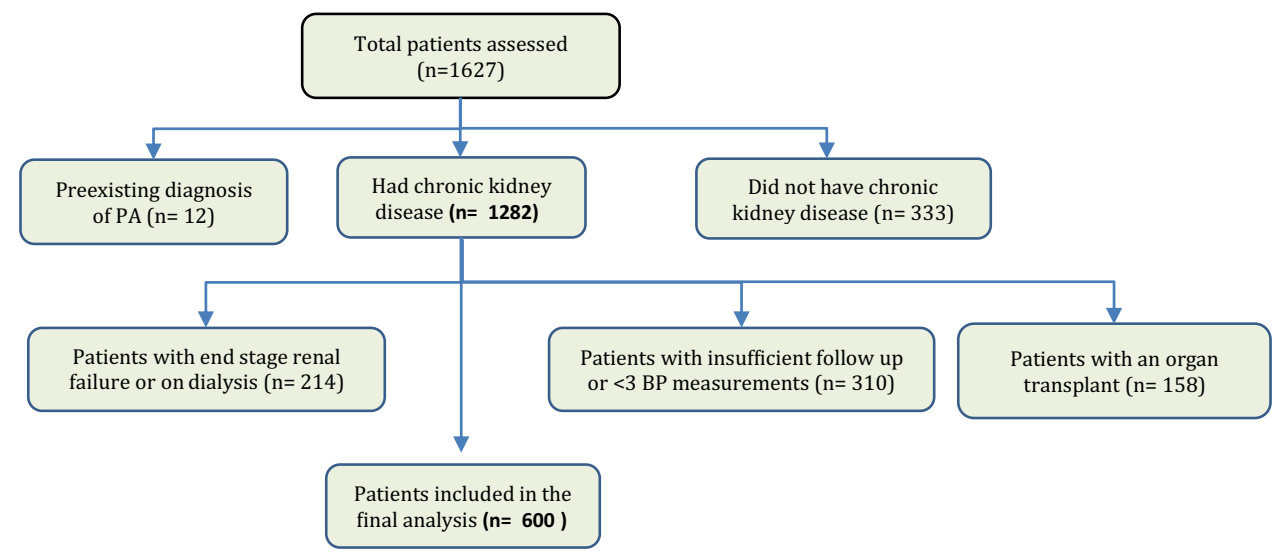

are presented in Table 2. However, of these 234 patients in whom PA screening was indicated, only 33 (14\% of 234) were screened for PA, including 21 (17\% of 122) at Monash Health and 12 (11\% of 112) at Alfred Health.

The most common indications for PA screening in our cohort related to the degree of hypertension control, with resistant hypertension $(n=94)$, controlled hypertension on 4 or more agents $(n=70)$ and blood pressure greater than $150 / 100(n=46)$ being the top three indications.

Patients indicated for screening were significantly older with a median age of 72 years (IQR, 57-79) than patients who were not (median age 69 years; 95\% CI: 53, 78) and had a significantly higher mean systolic BP of $140 \mathrm{mmHg}$ (IQR, 130-150) compared to $129 \mathrm{mmHg}$ (IQR, 122-137) for those not indicated for screening (Table 2). As expected, they were also on more antihypertensive agents, had more co-morbidities and a greater prevalence of hypertension (100\%) than their non-indicated counterpart (73\%). There was no statistically significant difference in their gender distribution, duration of hypertension, smoking history, alcohol consumption, eGFR or potassium concentration. The prevalence of hypertension in the overall cohort was 83\% (268 / 300 patients from Monash Health and 230/ 300 from Alfred Health), with $46 \%$ of them being uncontrolled (BP $>140 / 90 \mathrm{mmHg}$ ). The most common comorbidities in patients with indications for PA screening were dyslipidaemia (59\%), type-2 diabetes mellitus (49\%), obesity (40\%) and a history of acute myocardial infarction (28\%).

Of the 234 patients who had indications for PA screening, 33 (14\%) were screened (Table 3 ). They were younger, with a median age of 58 years (IQR, 45-70) compared to those who were indicated but not screened (median age 72 years [IQR, 61-81]), and had a higher mean systolic BP (153 mmHg [IQR, 138-160] vs $140 \mathrm{mmHg}$ [IQR, 130, 147]) and diastolic BP (84 mmHg [IQR, 76-93] vs $77 \mathrm{mmHg}$ [IQR, 73-80]).

Patients who were screened also had better renal function (mean eGFR $51 \mathrm{~mL} / \mathrm{min} / 1.73 \mathrm{~m}^{2}$; [IQR, 29-61] vs eGFR $37 \mathrm{~mL} / \mathrm{min} / 1.73 \mathrm{~m}^{2}$; [IQR, 27-48]), and lower mean serum potassium (4.1 mEq/L [IQR, 3.8-4.5] vs $4.5 \mathrm{mEq} / \mathrm{L}$; [IQR, 4.1-4.9]). The most common documented causes of CKD were diabetes mellitus (31\%), followed by hypertension $(28 \%)$ and glomerulonephritis $(15 \%)$. There was no difference in the aetiology of CKD between those who were screened and those who were not. Among the 33 patients screened for PA, 8 had an elevated ARR and 4 were diagnosed with PA. The remaining four were classified as "undefined" as they either did not undergo confirmatory testing or were lost to follow-up. Nine patients were incidentally found to have an adrenal adenoma during CT imaging as part of their renal workup. Three of these patients subsequently were tested with an ARR and a significantly high level was detected in one of them. Confirmatory testing was not performed for this particular subject and therefore a conclusive diagnosis of PA could not be established. Of the four diagnosed patients, two were biochemically and clinically cured with an adrenalectomy while two were treated with spironolactone for bilateral disease. Their eGFR decreased following targeted treatment but stabilised after 6-12 months, with baseline eGFR of $33,54,22$ and $64 \mathrm{~mL} / \mathrm{min} / 1.73 \mathrm{~m}^{2}$ and post-treatment eGFR of $41,47,16$ and $39 \mathrm{~mL} / \mathrm{min} / 1.73 \mathrm{~m}^{2}$, respectively at 12 months follow-up. The 2 patients treated medically had a suboptimal reduction in blood pressure and in the absence of renin measurements, we cannot assess whether their mineralocorticoid receptor antagonist dosage was adequate. Of the patients who were screened with an ARR, 88\% (29/33) were on interfering medications. These included dihydropyridine calcium channel blockers (taken by $71 \%$ ), angiotensin receptor blockers (taken by $37 \%$ ), angiotensin converting enzyme inhibitors (taken by $22 \%$ ) which can cause false negative results and beta blockers (taken by 38\%) which can cause false positive results (Supplementary Table 1). We did not find statistically significant differences in the doses of interfering antihypertensive medications taken between those who had a positive and negative ARR (data not shown). 
Table 2 Baseline characteristics of patients indicated for screening vs not indicated

\begin{tabular}{|c|c|c|c|}
\hline Patient characteristics & $\begin{array}{l}\text { Indicated for Screening } \\
(n=234)\end{array}$ & $\begin{array}{l}\text { Not indicated for screening } \\
(n=366)\end{array}$ & P-value \\
\hline Male/ Females & $144 / 90$ & $207 / 159$ & 0.227 \\
\hline Median age, years & $72(57,79)$ & $69(53,78)$ & 0.059 \\
\hline BMI, $\mathrm{kg} / \mathrm{m}^{2}$ & $30(26,34)$ & $28(24,32)$ & $<0.001$ \\
\hline $\mathrm{SBP}, \mathrm{mmHg}$ & $140(130,150)$ & $129(122,137)$ & $<0.001$ \\
\hline DBP, $\mathrm{mmHg}$ & $77(73,81)$ & $75(70,80)$ & $<0.001$ \\
\hline History of HTN, (\%) & $234(100 \%)$ & $268(73 \%)$ & $<0.001$ \\
\hline Duration of HTN, years & $10.7(9.4,12.0)$ & $10.1(8.9,11.4)$ & 0.541 \\
\hline Alcohol consumption & & & 0.316 \\
\hline Never & $110(52 \%)$ & $191(58 \%)$ & \\
\hline Moderate & $94(44 \%)$ & $124(38 \%)$ & \\
\hline Heavy & $8(4 \%)$ & $13(4 \%)$ & \\
\hline Smoking history & & & 0.623 \\
\hline Never & $137(62 \%)$ & $222(64 \%)$ & \\
\hline Past smoker & $59(27 \%)$ & $94(27 \%)$ & \\
\hline Current smoker & $26(12 \%)$ & $32(9 \%)$ & \\
\hline $\mathrm{eGFR}, \mathrm{mL} / \mathrm{min} / 1.73 \mathrm{~m}^{2}$ & $38(28,50)$ & $42(29,55)$ & 0.117 \\
\hline Creatinine, $\mathrm{mmol} / \mathrm{L}$ & $142(114,190)$ & $134(103,177)$ & 0.024 \\
\hline Potassium, mmol/L & $4.4(4.0,4.9)$ & $4.4(4.1,4.8)$ & 0.757 \\
\hline Number of Antihypertensive & $3(2,4)$ & $1(1,2)$ & $<0.001$ \\
\hline ACEi & $77(33 \%)$ & $87(24 \%)$ & 0.014 \\
\hline $\mathrm{ARB}$ & $93(40 \%)$ & $109(30 \%)$ & 0.012 \\
\hline Beta-blockers & $125(53 \%)$ & $90(25 \%)$ & $<0.001$ \\
\hline MRA & $36(15 \%)$ & $23(6 \%)$ & $<0.001$ \\
\hline Diuretics & $128(55 \%)$ & $96(26 \%)$ & $<0.001$ \\
\hline DHP CCB & $147(63 \%)$ & $93(25 \%)$ & $<0.001$ \\
\hline Non DHP CCB & $12(5 \%)$ & $8(2 \%)$ & 0.050 \\
\hline History of T2DM & $119(51 \%)$ & $130(36 \%)$ & $<0.001$ \\
\hline History of IHD & $68(29 \%)$ & $73(20 \%)$ & 0.011 \\
\hline History of CCF & $35(15 \%)$ & $30(8 \%)$ & 0.010 \\
\hline History of OSA & $59(25 \%)$ & $7(2 \%)$ & $<0.001$ \\
\hline History of CVA & $33(14 \%)$ & $32(9 \%)$ & 0.041 \\
\hline Adrenal Incidentaloma & $9(4 \%)$ & $0(0 \%)$ & $<0.001$ \\
\hline $\begin{array}{l}\text { Family history of hypertension/ } \\
\text { stroke }<40 \text { years of age }\end{array}$ & $12(5 \%)$ & $1(0.3 \%)$ & $<0.001$ \\
\hline
\end{tabular}

$B M I$ body mass index, $S B P$ systolic blood pressure, $D B P$ diastolic blood pressure, eGFR estimated glomerular filtration rate, $A C E i$ angiotensin-converting enzyme inhibitor, $A R B$ angiotensin receptor blocker, $M R A$ mineralocorticoid receptor antagonist, $D H P C C B$ dihydropyridine calcium channel blocker, $T 2 D M$ type-2 diabetes mellitus, $I H D$ ischaemic heart disease, $C C F$ congestive cardiac failure, $O S A$ obstructive sleep apnoea, CVA cerebrovascular accident; normal levels for eGFR: $>90 \mathrm{~mL} / \mathrm{min} / 1.73 \mathrm{~m}^{2}$; normal levels for creatinine: $60-110 \mathrm{mmol} / \mathrm{L}$; normal levels for serum potassium: $3.5-5.0 \mathrm{mmol} / \mathrm{L}$

\section{Discussion}

To our knowledge, this is the first study to evaluate the pattern of screening for PA as a cause of secondary hypertension in patients with CKD. Our patient cohort is representative of a typical CKD population [34] with the most common causes of CKD being diabetes mellitus, glomerulonephritis and hypertension. The prevalence of hypertension in our cohort was $83 \%$, which is consistent with the findings of global data analysis [35].

We observed that $39 \%$ of patients had indications for PA testing at their first presentation to the nephrology clinic based on the guidelines set out by the Endocrine Society. However, less than $14 \%$ of patients who met the criteria for screening were actually tested for PA. While these rates are low, they are higher than those reported in prior studies of hypertensive groups without CKD [25, 26, 31]. 
Table 3 Baseline characteristics of patients with indications for screening

\begin{tabular}{|c|c|c|c|}
\hline Patient characteristics & $\begin{array}{l}\text { Indicated and screened } \\
(\mathrm{n}=33)\end{array}$ & $\begin{array}{l}\text { Indicated but not screened } \\
(\mathrm{n}=201)\end{array}$ & P-value \\
\hline Male/ Females & $23 / 10$ & $121 / 80$ & 0.223 \\
\hline Median age, years & $58(45,70)$ & $72(61,81)$ & $<0.001$ \\
\hline BMI, $\mathrm{kg} / \mathrm{m}^{2}$ & $30(26,33)$ & $30(26,34)$ & 0.922 \\
\hline $\mathrm{SBP}, \mathrm{mmHg}$ & $153(138,160)$ & $140(130,147)$ & $<0.001$ \\
\hline $\mathrm{DBP}, \mathrm{mmHg}$ & $84(76,93)$ & $77(73,80)$ & $<0.001$ \\
\hline History of HTN, (\%) & $33(100 \%)$ & $201(100 \%)$ & 0.360 \\
\hline Duration of HTN, years & $11.5(6.7,16.3)$ & $9.7(8.5,11)$ & 0.443 \\
\hline Alcohol consumption & & & 0.835 \\
\hline Never & $16(57 \%)$ & $94(51 \%)$ & \\
\hline Moderate & $11(39 \%)$ & $83(45 \%)$ & \\
\hline Heavy & $1(4 \%)$ & $7(4 \%)$ & \\
\hline Smoking history & & & 0.173 \\
\hline Never & $17(61 \%)$ & $120(62 \%)$ & \\
\hline Past smoker & $5(18 \%)$ & $54(28 \%)$ & \\
\hline Current smoker & $6(21 \%)$ & $20(10 \%)$ & \\
\hline $\mathrm{eGFR}, \mathrm{mL} / \mathrm{min} / 1.73 \mathrm{~m}^{2}$ & $51(29,61)$ & $37(27,48)$ & 0.041 \\
\hline Creatinine, $\mathrm{mmol} / \mathrm{L}$ & $128(94,204)$ & $143(117,191)$ & 0.378 \\
\hline Potassium, mmol/L & $4.1(3.8,4.5)$ & $4.5(4.1,4.9)$ & 0.009 \\
\hline Number of Antihypertensive & $3(2,4)$ & $3(2,4)$ & 0.937 \\
\hline $\mathrm{ACEi}$ & $9(27 \%)$ & $68(34 \%)$ & 0.457 \\
\hline $\mathrm{ARB}$ & $11(33 \%)$ & $82(41 \%)$ & 0.417 \\
\hline Beta blocker & $14(42 \%)$ & $111(55 \%)$ & 0.172 \\
\hline MRA & $3(9 \%)$ & $33(16 \%)$ & 0.280 \\
\hline Diuretics & $14(42 \%)$ & $114(57 \%)$ & 0.308 \\
\hline DHP CCB & $24(73 \%)$ & $123(62 \%)$ & 0.216 \\
\hline Non DHP CCB & $3(9 \%)$ & $9(5 \%)$ & 0.265 \\
\hline History of T2DM & $10(30 \%)$ & $109(54 \%)$ & 0.011 \\
\hline History of IHD & $5(15 \%)$ & $63(31 \%)$ & 0.058 \\
\hline History of CCF & $3(9 \%)$ & $32(16 \%)$ & 0.308 \\
\hline History of OSA & $7(21 \%)$ & $52(26 \%)$ & 0.568 \\
\hline History of CVA & $7(21 \%)$ & $26(13 \%)$ & 0.205 \\
\hline Adrenal Incidentaloma & $3(9 \%)$ & $6(3 \%)$ & 0.091 \\
\hline $\begin{array}{l}\text { Family history of hypertension/ } \\
\text { stroke }<40 \text { years of age }\end{array}$ & $5(15 \%)$ & $7(3 \%)$ & 0.005 \\
\hline
\end{tabular}

$B M I$ body mass index, $S B P$ systolic blood pressure, $D B P$ diastolic blood pressure, eGFR estimated glomerular filtration rate, $A C E i$ angiotensin-converting enzyme inhibitor, $A R B$ angiotensin receptor blocker, $D H P C C B$ dihydropyridine calcium channel blocker, T2DM type-2 diabetes mellitus, IHD ischaemic heart disease, $C C F$ congestive cardiac failure, $O S A$ obstructive sleep apnoea, $C V A$ cerebrovascular accident; normal levels for eGFR: $>90 \mathrm{~mL} / \mathrm{min} / 1.73 \mathrm{~m}^{2}$; normal levels for creatinine: $60-110 \mathrm{mmol} / \mathrm{L}$; normal levels for serum potassium: $3.5-5.0 \mathrm{mmol} / \mathrm{L}$
Previous studies have also found that consultations with a nephrologist or endocrinologist were independently associated with a higher likelihood of screening as compared to cardiologists or general practitioners [36]. Therefore, due to the involvement of dedicated nephrologists at both study sites and the presence of an Endocrine Hypertension Service at Monash Health, it is likely that the screening rate seen in this study is an overestimate of PA screening in the wider CKD population, a large proportion of whom are managed at a primary care level [37].

We observed that a lower serum potassium concentration was independently associated with a greater likelihood of screening for PA. This is likely due to the classic presentation of PA as hypertension with hypokalemia. However, a large proportion of patients with PA have normal potassium concentrations. A study by Rossi et al. in the general 
population found that only $52 \%$ of patients with an aldosterone-producing adenoma and $17 \%$ of patients with bilateral adrenal hyperplasia were hypokalaemic [38], highlighting the importance of testing for PA even if potassium concentration is within normal range. One might expect this point to be even more significant for the CKD population who have a propensity for hyperkalaemia because of their underlying renal impairment. Patients with a higher eGFR and younger age were also more likely to be screened for PA. This is possibly due to the perceived benefit of PA treatment being greater in patients with less severe disease and to limited guidelines for the interpretation of aldosterone and renin levels in patients with CKD.

In keeping with other studies that highlighted delays in the diagnosis of PA [39], our study also found the median duration of hypertension to be 10.7 years in patients with indications for screening. As a result, $69 \%$ of these patients were on 3 or more antihypertensives at the time of their screening test. This complicated the interpretation of their ARR due to the effect these medications have on aldosterone and renin levels [40].

The Endocrine Society recommends that all medications that interfere with the ARR be withdrawn, in particular, mineralocorticoid receptor antagonists and other diuretics should be ceased at least 4 weeks before ARR testing [24]. A complete washout of all interfering medications or substitution with non-interfering agents is also recommended. However, due to the complexity of managing CKD, there may be a reluctance to change medications such as angiotensin receptor blockers and angiotensin converting enzyme inhibitors due to comorbid heart disease or diabetes. Additionally, re-testing after a period of washout may not always be feasible due to the time constraints encountered commonly in outpatient settings. A previous study even suggested alternative ARR thresholds for patients on interfering medications [41]. However, due to the retrospective nature of our study, we do not have enough data to comment on the necessity to withdraw interfering antihypertensive medications. This is clearly relevant as $88 \%$ of patients who underwent screening were on interfering medications. Whilst the ARR can be interpreted in the context of these confounding factors [34], the possibility of a negative screening test is rarely followed up with additional testing. The effects of different antihypertensive medications on aldosterone, renin and the aldosterone-to-renin ratio with tips on interpreting these results have been described in Table 4 [42].

A failure to screen for PA in indicated patients may reflect inadequate awareness of the disease or a general proclivity for treatment inertia since hypertension is both a common cause and complication of CKD [43]. The commonly used confirmatory test which requires the infusion of $2 \mathrm{~L}$ saline over $4 \mathrm{~h}$ may be considered a relative contraindication in patients with severe CKD and fluid overload, and therefore serve as a deterrent to adequate PA testing. Oral salt loading poses the same risk and requires $24-\mathrm{h}$ urine collection which may be inaccurate in the context of reduced urine output [44]. The best alternative test is the captopril challenge test, but it requires further validation in Caucasian populations. Ideally, patients are tested before they develop moderate to severe CKD. Another possible consideration for deficits in screening and treatment of PA in patients with CKD is the expected decline in eGFR which reflects the reversal of glomerular hyperfiltration seen in untreated hyperaldosteronism [14]. This reversal is presumed to be due to a combination

Table 4 Effects of antihypertensives on aldosterone, renin and aldosterone-to-renin ratio

\begin{tabular}{|c|c|c|c|c|}
\hline Drug & $\begin{array}{l}\text { Effect on } \\
\text { aldoster- } \\
\text { one }\end{array}$ & Effect on renin & Effect on ARR & Interpretation \\
\hline ACEi & $\downarrow$ & $\uparrow \uparrow$ & $\downarrow$ & $\begin{array}{l}\text { Low renin is a strong predictor of PA. High renin does not } \\
\text { exclude it }\end{array}$ \\
\hline ARB & $\downarrow$ & $\uparrow \uparrow$ & $\downarrow$ & Same as for ACE inhibitors \\
\hline Beta-blocker & $\downarrow$ & $\downarrow \downarrow$ & $\uparrow$ & Increased ARR is not clinically relevant if aldosterone is low \\
\hline Calcium channel blocker (DHP) & $\leftrightarrow \downarrow$ & $\leftrightarrow \uparrow$ & $\downarrow$ & Discontinuation before testing is recommended \\
\hline Verapamil & $\leftrightarrow$ & $\leftrightarrow$ & $\leftrightarrow$ & Considered non-interfering \\
\hline Prazosin & $\leftrightarrow$ & $\leftrightarrow$ & $\leftrightarrow$ & Considered non-interfering \\
\hline Moxonidine & $\leftrightarrow$ & $\leftrightarrow$ & $\leftrightarrow$ & Considered non-interfering \\
\hline Hydralazine & $\leftrightarrow$ & $\leftrightarrow$ & $\leftrightarrow$ & Considered non-interfering \\
\hline MRA & $\leftrightarrow \uparrow$ & $\leftrightarrow \uparrow \uparrow$ & $\leftrightarrow \downarrow$ & $\begin{array}{l}\text { Diagnosis of PA can be made in patients with high aldosterone } \\
\text { and low renin. If renin is not supressed, discontinuation for } \\
4-6 \text { weeks is recommended before retesting }\end{array}$ \\
\hline Potassium wasting diuretic & $\leftrightarrow \uparrow$ & $\uparrow \uparrow$ & $\downarrow$ & Discontinuation for $4-6$ weeks is recommended before testing \\
\hline
\end{tabular}

$A C E i$ angiotensin-converting enzyme inhibitor, $A R B$ angiotensin receptor blocker, $D H P$ dihydropyridine, $M R A$ mineralocorticoid receptor antagonist 
of decreased BP and blockade of aldosterone-mediated hyperfiltration [45]. A study of 600 patients with treated PA demonstrated that surgical treatment was better at preventing long-term renal dysfunction than mineralocorticoid receptor antagonist therapy [19]. A recent trial by Bakris et al. found that treatment of type- 2 diabetic CKD patients with finerenone, a non-steroidal, selective mineralocorticoid receptor antagonist resulted in lower risks of CKD progression and cardiovascular events [46]. However, there has also been conflicting evidence on the recommendation for use of spironolactone and other mineralocorticoid receptor antagonists in end-stage renal disease [15]. The aforementioned factors coupled with a lack of specific guidelines for diagnosing PA in patients with CKD may explain the low rate of screening. However, the exact barriers to testing for PA need to be examined in prospective studies to develop best practice guidelines in the CKD clinic.

The strengths of this study include the large, well-characterised sample of patients with CKD across two tertiary referral centres. They are representative of the typical CKD cohort based on the causes of their renal disease and prevalence of hypertension. The primary limitation of our study is the retrospective observational design resulting in the exclusion of 310 of 1627 patients due to inadequate follow-up. Of the included patients who were diagnosed with PA, few had adequate biochemical follow-up with aldosterone and renin measurements to evaluate the success of their treatment. Two patients who were treated medically for bilateral disease had a suboptimal reduction in blood pressure but the adequacy of treatment could not be assessed in the absence of follow-up renin measurements. All patients included in the analysis were referred to specialised nephrology clinics which could potentially limit the generalisability of our results to the wider CKD population, many of whom are managed within primary care [40]. For most patients, their hypertensive status and control was assessed through inclinic BP measurements which may be prone to a greater degree of variability than standardised 24-h ambulatory blood pressure measurements. For patients who underwent screening for PA, plasma aldosterone concentration was measured using chemiluminescent immunoassays which may give falsely high values in patients with chronic renal disease due to accumulating cross-reacting steroid metabolites $[47,48]$. Furthermore, patients with CKD have been found to have increasing plasma aldosterone and renin concentrations as creatinine clearance declines which could confound the interpretation of the ARR in this patient group [49, 50]. However, as demonstrated in Supplementary Table 1, patients who had ARR above the threshold of $70 \mathrm{pmol} /$ mU had similar eGFR as those with ARR below $70 \mathrm{pmol} /$ $\mathrm{mU}$, which reinforces the clinical usefulness of the ARR as a screening tool even in the CKD population. This discrepancy could be evaluated in future studies by measuring aldosterone with liquid chromatography-tandem mass spectrometry assays (LC-MS/MS) which have greater precision than immunoassays [47]. Finally, scanned medical records were manually assessed and all steps were taken to include the results of testing performed outside the health network if available. Any testing that was not formally documented with the treating team would have been missed, although it is unlikely that patients were evaluated for PA without the knowledge of their nephrologists.

\section{Conclusion}

In summary, this study found that the rate of screening for primary aldosteronism, based on Endocrine Society guidelines, was low in a CKD population, especially in patients who were older, had lower eGFR and normal serum potassium. The consequences of undiagnosed primary aldosteronism in this population may be substantial due to the cardiovascular and renal sequelae associated with untreated disease. Given that $46 \%$ of our cohort had uncontrolled hypertension, it would appear necessary to screen all of these patients for primary aldosteronism with the aldosterone to renin ratio so as to facilitate the timely diagnosis of a common, treatable and potentially curable form of hypertension. A prospective study is needed to evaluate the outcomes of routine primary aldosteronism screening in patients with CKD to both understand the prevalence of primary aldosteronism and the blood pressure and renal outcomes of targeted treatment [51].

Supplementary Information The online version contains supplementary material available at https://doi.org/10.1007/s40620-022-01267-3.

Acknowledgements The Hudson Institute is supported by the State Government of Victoria's Operational Infrastructure Support Program. JY is supported by a National Health and Medical Research Council (NHMRC) Investigator Grant and RL is supported by a NHMRC Postgraduate Scholarship.

Author contributions All co-authors were involved in designing the study and revising the article critically for important intellectual content. E.S and K.C. performed data collection. R.L. analysed and interpreted the collected data. K.C and J.Y. drafted the manuscript. All the authors have approved the final version of the article to be published and agreed to be accountable for all aspects of the work.

Funding Open Access funding enabled and organized by CAUL and its Member Institutions. No funds, grants, or other support was received.

Data availability statement The datasets generated and/or analysed during the current study are available from the corresponding author JY on reasonable request. The data are not publicly available due to them containing confidential patient information. 


\section{Declarations}

Conflict of interest The authors have no relevant financial or non-financial interests to disclose. The results presented in this paper have not been published elsewhere.

Ethical approval This research study was conducted retrospectively from data obtained for clinical purposes. This was done in line with the principles of the 1964 Helsinki Declaration. Approval was granted by the Human Research Ethics Committees of Monash Health and Alfred Health (Australia). The confidentiality of the data was maintained during the study.

Open Access This article is licensed under a Creative Commons Attribution 4.0 International License, which permits use, sharing, adaptation, distribution and reproduction in any medium or format, as long as you give appropriate credit to the original author(s) and the source, provide a link to the Creative Commons licence, and indicate if changes were made. The images or other third party material in this article are included in the article's Creative Commons licence, unless indicated otherwise in a credit line to the material. If material is not included in the article's Creative Commons licence and your intended use is not permitted by statutory regulation or exceeds the permitted use, you will need to obtain permission directly from the copyright holder. To view a copy of this licence, visit http://creativecommons.org/licenses/by/4.0/.

\section{References}

1. Head GA, Shaw JE, Dunstan DW et al (2019) Hypertension, white-coat hypertension and masked hypertension in Australia: findings from the Australian Diabetes, Obesity, and Lifestyle Study 3. Am J Hypertens 37(8):1615-1623

2. De Nicola L, Zoccali C (2016) Chronic kidney disease prevalence in the general population: heterogeneity and concerns. Nephrol Dial Transplant 31(3):331-335

3. Ku E, Lee BJ, Wei J, Weir MR (2019) Hypertension in CKD: core curriculum 2019. Am J Kidney Dis 74(1):120-131

4. Gordon RD (1995) Primary aldosteronism. J Endocrinol Invest 18(7):495-511

5. Ganguly A (1998) Primary aldosteronism. N Engl J Med 339(25):1828-1834

6. Tiu S-C, Choi C-H, Shek C-C et al (2005) The use of aldosteronerenin ratio as a diagnostic test for primary hyperaldosteronism and its test characteristics under different conditions of blood sampling. J Clin Endocrinol Metab 90(1):72-78

7. Montori VM, Young Jr W (2002) Use of plasma aldosterone concentration-to-plasma renin activity ratio as a screening test for primary aldosteronism. A systematic review of the literature. Endocrinol Metab Clin N Am. 31(3):619-632 (xi)

8. Rossi G-P, Sechi LA, Giacchetti G, Ronconi V, Strazzullo P, Funder JW (2008) Primary aldosteronism: cardiovascular, renal and metabolic implications. Trends Endocrinol Metab 19(3):88-90

9. Mattsson C, Young WF (2006) Primary aldosteronism: diagnostic and treatment strategies. Nat Rev Nephrol 2(4):198-208

10. Mulatero P, Monticone S, Bertello C et al (2013) Long-term cardio- and cerebrovascular events in patients with primary aldosteronism. J Clin Endocrinol Metab 98(12):4826-4833

11. Reincke M, Fischer E, Gerum S et al (2012) Observational study mortality in treated primary aldosteronism: the German Conn's registry. Hypertension 60(3):618-624
12. Monticone S, D'Ascenzo F, Moretti C et al (2018) Cardiovascular events and target organ damage in primary aldosteronism compared with essential hypertension: a systematic review and meta-analysis. Lancet Diabetes Endocrinol 6(1):41-50

13. Milliez P, Girerd X, Plouin P-F, Blacher J, Safar ME, Mourad J-J (2005) Evidence for an increased rate of cardiovascular events in patients with primary aldosteronism. J Am Coll Cardiol 45(8): $1243-1248$

14. Ribstein J, Cailar GD, Fesler P, Mimran A (2005) Relative glomerular hyperfiltration in primary aldosteronism. JASN 16(5):1320-1325

15. Sechi LA, Novello M, Lapenna R et al (2006) Long-term renal outcomes in patients with primary aldosteronism. JAMA 295(22):2638-2645

16. Ogata H, Yamazaki Y, Tezuka Y, et al (2021) Renal injuries in primary aldosteronism: quantitative histopathological analysis of 19 patients with primary adosteronism. Hypertension, $p$ HYPERTENSIONAHA-121.

17. Utsumi T, Kawamura K, Imamoto T et al (2013) Preoperative masked renal damage in Japanese patients with primary aldosteronism: Identification of predictors for chronic kidney disease manifested after adrenalectomy. Int J Urol 20(7):685-691

18. Wu V-C, Wang S-M, Chang C-H, Hu Y-H, Lin L-Y, Lin Y-H et al (2016) Long term outcome of Aldosteronism after target treatments. Sci Rep 6(1):32103

19. Hundemer GL, Curhan GC, Yozamp N, Wang M, Vaidya A (2018) Renal outcomes in medically and surgically treated primary aldosteronism. Hypertension 72(3):658-666

20. Rossi GP, Maiolino G, Flego A et al (2018) Adrenalectomy lowers incident atrial fibrillation in primary aldosteronism patients at long term. Hypertension 71(4):585-591

21. Lubitz CC, Economopoulos KP, Sy S et al (2015) Cost-effectiveness of screening for primary aldosteronism and subtype diagnosis in the resistant hypertensive patients. Circ Cardiovasc Qual Outcomes 8(6):621-630

22. Conn JW (1955) Presidential address. I. Painting background. II. Primary aldosteronism, a new clinical syndrome. J Lab Clin Med 45(1):3-17

23. Käyser SC, Dekkers T, Groenewoud HJ et al (2016) Study heterogeneity and estimation of prevalence of primary aldosteronism: a systematic review and meta-regression analysis. J Clin Endocrinol Metab 101(7):2826-2835

24. Funder JW, Carey RM, Mantero F et al (2016) The management of primary aldosteronism: case detection, diagnosis, and treatment: an Endocrine Society Clinical Practice Guideline. J Clin Endocrinol Metab 101(5):1889-1916

25. Jaffe G, Gray Z, Krishnan G et al (2020) Screening rates for primary aldosteronism in resistant hypertension. Hypertension 75(3):650-659

26. Liu Y, King J, Kline GA et al (2021) Outcomes of a specialized clinic on rates of investigation and treatment of primary aldosteronism. JAMA 156(6):541-549

27. Wu J, Tian W, Zhang L, Zhang J, Zhou B (2019) Assessing the quality of guidelines for primary aldosteronism: which guidelines are worth applying in diverse settings? J Hypertens 37(7): 1500

28. Huang Q-F, Hoshide S, Cheng H-M et al (2016) Management of hypertension in patients with chronic kidney disease in Asia. Curr Hypertens Rev 12(3):181-185

29. National Heart Foundation of Australia, National Blood Pressure and Vascular Disease Advisory Committee (2016) Guideline for the diagnosis and management of hypertension in adults

30. Cheung AK, Chang TI, Cushman WC et al (2021) KDIGO 2021 clinical practice guideline for the management of blood pressure in chronic kidney disease. Kidney Int 99(3):S1-87 
31. Sivarajah M, Beninato T, Fahey TJ (2020) Adherence to consensus guidelines for screening of primary aldosteronism in an urban healthcare system. Surgery 167(1):211-215

32. Stowasser M, Ahmed AH, Pimenta E, Taylor PJ, Gordon RD (2012) Factors affecting the aldosterone/renin ratio. Horm Metab 44(03):170-176

33. Ahmed AH, Gordon RD, Taylor PJ, Ward G, Pimenta E, Stowasser M (2011) Effect of contraceptives on aldosterone/renin ratio may vary according to the components of contraceptive, renin assay method, and possibly route of administration. J Clin Endocrinol Metab 96(6):1797-1804

34. Evans PD, Taal MW (2011) Epidemiology and causes of chronic kidney disease. Medicine 39(7):402-406

35. Kearney PM, Whelton M, Reynolds K, Muntner P, Whelton PK, He J (2005) Global burden of hypertension: analysis of worldwide data. The Lancet 365(9455):217-223

36. Cohen JB, Cohen DL, Herman DS, Leppert JT, Byrd JB, Bhalla V (2021) Testing for primary aldosteronism and mineralocorticoid receptor antagonist use among U.S. veterans. Ann Intern Med 174(3):289-297

37. Gelder VAV, Haan NDS-D, Grauw WJCD et al (2016) Quality of chronic kidney disease management in primary care: a retrospective study. Scand J Prim Health Care 34(1):73-80

38. Rossi GP, Bernini G, Caliumi $\mathrm{C}$ et al (2006) A prospective study of the prevalence of primary aldosteronism in 1,125 hypertensive patients. J Am Coll Cardiol 48(11):2293-2300

39. Lim YY, Shen J, Fuller PJ, Yang J (2018) Current pattern of primary aldosteronism diagnosis: delayed and complicated. Aust J Gen Pract 47(10):712-718

40. Mulatero P, Rabbia F, Milan A et al (2002) Drug effects on aldosterone/plasma renin activity ratio in primary aldosteronism. Hypertension 40(6):897-902

41. O'Shea PM, Griffin TP, Denieffe S, Fitzgibbon MC (2019) The aldosterone to renin ratio in the diagnosis of primary aldosteronism: promises and challenges. Int J Clin 73(7):e13353

42. Jędrusik P, Symonides B, Lewandowski J, Gaciong Z (2021) The effect of antihypertensive medications on testing for primary aldosteronism. Front Pharmacol 12
43. Horowitz B, Miskulin D, Zager P (2015) Epidemiology of hypertension in CKD. Adv Chronic Kidney Dis 22(2):88-95

44. Ergin AB, Hamrahian AH, Kennedy AL, Gupta MK (2015) Intravenous Saline suppression test. In: The Cleveland Clinic manual of dynamic endocrine testing. Springer, Cham, pp 59-62

45. Kuo C-C, Wu V-C, Tsai C-W, Wu K-D (2011) Relative kidney hyperfiltration in primary aldosteronism: a meta-analysis. J Renin Angiotensin Aldosterone Syst 12(2):113-122

46. Bakris GL, Agarwal R, Anker SD, Pitt B, Ruilope LM, Rossing $P$ et al (2020) Effect of finerenone on chronic kidney disease outcomes in type 2 diabetes. N Engl J Med 383(23):2219-2229

47. Koshida H, Miyamori I, Miyazaki R, Tofuku Y, Takeda R (1989) Falsely elevated plasma aldosterone concentration by direct radioimmunoassay in chronic renal failure. J Lab Clin Med 114(3):294-300

48. Lam L, Chiu WW, Davidson JS (2016) Overestimation of aldosterone by immunoassay in renal impairment. Clin Chem 62(6):890-891

49. Hené RJ, Boer P, Koomans HA, Mees EJ (1982) Plasma aldosterone concentrations in chronic renal disease. Kidney Int 21(1):98-101

50. Weidmann P, Maxwell MH, Lupu AN, Lewin AJ, Massry SG (1971) Plasma renin activity and blood pressure in terminal renal failure. NEJM 285(14):757-762

51. Thomas G, Xie D, Chen H-Y et al (2016) Prevalence and prognostic significance of apparent treatment resistant hypertension in chronic kidney disease. Hypertension 67(2):387-396

Publisher's Note Springer Nature remains neutral with regard to jurisdictional claims in published maps and institutional affiliations. 